\title{
von Willebrand Disease, A Rare Cause of Massive Upper GI Bleeding: A Case Report
}

\author{
RAFIA RASHID ${ }^{1}$, MD WAHIDUZZAMAN MAZUMDER ${ }^{2}$, ASM BAZLUL KARIM ${ }^{3}$, FAHMIDA BEGUM ${ }^{4}$, \\ FAHMIDA ISLAM ${ }^{5}$, ZANNATUL FERDOUS SONIA ${ }^{5}$
}

\begin{abstract}
von Willebrand Disease (VWD) is the most common inherited bleeding disorder. It occurs due to either qualitative or quantitative defect of von Willebrand Factor (VWF). VWF is a large multimeric glycoprotein necessary for platelet aggregation and adhesion to the subendothelium following any vascular injury. Typical presentation of VWD patient include mucosal bleeding, easy bruising and bleeding following minor trauma. Rarely, these patients may present with gastro intestinal bleeding. The treatment of choice in mild forms of VWD is the synthetic agent desmopressin. In patients with severe form or in children who do not response to desmopressin, the appropriate treatment is a VWF rich factor VIII concentrate. There are few case reports of VWD with upper gastrointestinal (UGI) bleeding. So, we report a case of 9 years old female child who was admitted at Bangabandhu Sheikh Mujib Medical University in Paediatric Gastroenterology Department with the complaints of recurrent UGI bleeding for 3 years. She also had history of spontaneous gum bleeding, easy bruising, echymosis, prolong bleeding following minor trauma since early age. After appropriate evaluation of the patient including history and relevant investigation, she was diagnosed as a case of VWD with GI bleeding. We report this as it is a rare presentation of VWD.
\end{abstract}

Key words: Upper GI bleeding, von Willebrand Disease

\section{Introduction}

Upper gastrointestinal (GI) bleeding in children is not so uncommon. Bleeding from the gastrointestinal tract (GIT) can be divided into: Upper GI (UGI) bleeding and Lower GI (LGI) bleeding. UGI bleeding is defined as the bleeding from a lesion in the GIT proximal to the ligament of Treitz. UGI bleeding is usually presents as hematemesis and melena. Hematemesis can be defined as vomiting that

1. Post Graduate Trainee, Department of Paediatric Gastroenterology and Nutrition, BSMMU, Dhaka

2. Associate Professor, Department of Paediatric Gastroenterology and Nutrition, BSMMU, Dhaka

3. Professor, Department of Paediatric Gastroenterology and Nutrition, BSMMU, Dhaka

4. Assistant Professor, Department of Paediatric Gastroenterology and Nutrition, BSMMU, Dhaka

5. Resident, Department of Paediatric Gastroenterology and Nutrition, BSMMU, Dhaka

Correspondence: Dr. Rafia Rashid, Department of Paediatric Gastroenterology and Nutrition, BSMMU, Dhaka. Cell no: 01819420570, email: reete2000@gmail.com

Received: 23/08/18 contains bright red blood and coffee ground material indicating a change of blood color by gastric acid. Melena can be defined as a dark colored tarry stool due to the effect of intestinal bacteria on the blood leading to oxidizing hemoglobin to hematin ${ }^{1}$. Rarely UGIB may present as hematochezia which is defined as fresh or bright red blood evacuating from the anus. ${ }^{1}$ In rare situations, UGIB may be due to underlying coagulation disorder like von Willebrand disease (VWD). Originally described by Erik von Willebrand in 1926, VWD is usually the most common human inherited bleeding disorder with a prevalence of $1: 100$ to $1 ; 10,000 .^{2}$

von Willebrand factor(VWF) is the largest human plasma protein, and an adhesive multimeric glycoprotein. VWF is synthesized in two cell types: one is vascular endothelium, and another is bone marrow megakaryocytes. In vascular endothelium VWF is stored in secretory granules (Weibel-Palade bodies) from which it can be released by stress or 
drugs such as desmopressin. In bone marrow megakaryocytes, it is stored in platelet alpha granules from which it is released following platelet activation. 3 VWF mediates platelet adhesion to both the subendothelial matrix and endothelial surfaces and acts as a carrier for coagulation factor VIII (FVIII) in the circulation during vascular injury which is essential for normal primary haemostasis. ${ }^{4}$ VWD is an autosomal (dominant and recessive) bleeding disorder that arises from deficiencies in VWF. The defect varies from quantitative to qualitative or even total deficiency of VWF. ${ }^{5}$ There are three main types of VWD: Type 1, 2 and 3. Type 1 (quantitative deficiency of VWF) is characterized by mild bleeding which is caused by a variety of genetic mutations in the VWF gene (which resides in chromosome 12). Type 2 VWD is characterized by qualitative defects in the VWF molecule which is relatively less common. Type 3 VWD is characterized by severe bleeding due to almost absence of VWF and pseudo von Willebrand, and it is a rarer form. ${ }^{6,7}$ VWD typically presents with mucosal bleeding, epistaxis, easy bruising, postpartum hemorrhage, joint bleeding, and gastrointestinal bleeding. 2,7,8 VWD patients exhibits a normal platelet count, normal clotting time, and normal prothrombin time, but the bleeding time is increased to an extremely variable degree. ${ }^{8} \mathrm{We}$ report the observation of a child admitted to the department of Paediatric Gastroenterology and Nutrition in BSMMU for a state of Haematemesis and melena revealing a severe defect in VWF.

\section{Case Report}

We present the case of a 9 year old female child. She was admitted in the department of Paediatric Gastroenterology and Nutrition in BSMMU with the complaints of profuse haematemesis and melena for 7 days. She had history of same type of attacks for three times in last three years. With these complaints she was admitted in different hospitals and received blood transfusions for 4 times. She had history of spontaneous gum bleeding, easy bruising, and prolonged bleeding following trauma since 2 years of her age. She is the first child of her parents. No history of parental consanguinity. Her birth history was unremarkable. None of her family members was suffering from bleeding disorder. The child was on a normal diet and was thriving well. She was properly vaccinated and there were no incidents during vaccination. Clinically the child was alert, cooperative, moderately pale, multiple bruises over extremities.
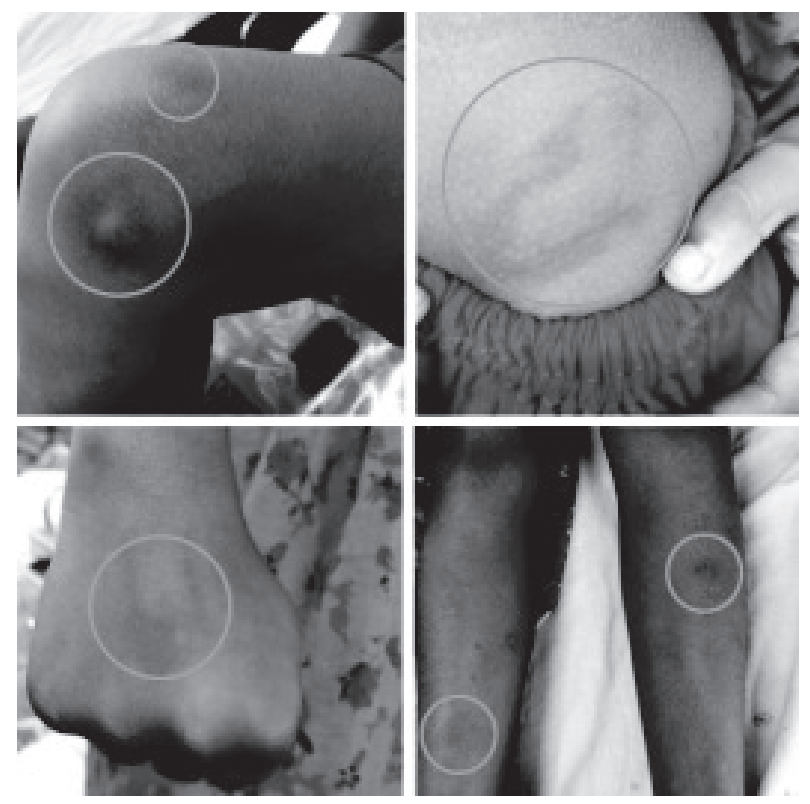

Fig.-1: Multiple bruises over extremities.

Her vitals were within normal limit except tachycardia, and oral cavity was normal. There was no organomegaly or joint swelling (Hemarthosis). On admission the child had no active bleeding, and only nutritional supplement was given. Laboratory investigations showed hemoglobin $(\mathrm{Hb})$ level at 8.8 $\mathrm{g} / \mathrm{dL}$ (normal value 11.5-14.5 g/dL), platelet count was normal, bleeding time (BT) was 10 minutes (normal value 2-7 minutes), clotting time (CT) was 15 minutes (normal value 5-8 minutes). Upper GI endoscopy was normal, and bone marrow study revealed normal active marrow. The result of Von Willebrand factor:Ag assay was very low at $1 \%$ (normal value $50 \%-160 \%$ ) that confirmed a severe form of Von Willebrand disease (type 3). After consultation with Paediatric Haematology and Oncology Department the child was treated with desmopressin acetate and tranexamic acid. Patient was discharged with advice of sedentary lifestyle in combination with prophylactic therapy in minor surgery including dental work. Desmopressin nasal spray was advised when necessary (if bleeding including haematemesis \& melena occurs).

\section{Discussion}

VWD is a complex genetic disorder due to qualitative and or quantitative deficiency of VWF. VWF is a large multimeric glycoprotein crucial for primary hemostasis in human. It is synthesized by endothelial cells and megakaryocytes, secreted into plasma, and 
their size is regulated by a specific cleaving-protease named ADAMTS13. The gene coding for VWF has been located at chromosome 12p13.2. ${ }^{9}$ The symptoms of VWD vary among patients, depending on the level of residual VWF activity, the disease subtype, and to some extent, age and sex. ${ }^{10}$ In children with VWD the most frequent presenting symptoms are bruising and epistaxis. ${ }^{10}$ Our presenting case had history of easy bruising, spontaneous gum bleeding, and prolonged bleeding following minor trauma since her early life. The majority of VWD patients (60 to $80 \%$ ) have bleeding after surgery or dental extractions. ${ }^{11}$ Our patient didn't have such history. In severe form (Type 3) VWD may presents with joint bleeds. ${ }^{1} \mathrm{~A}$ well-known, serious, and possibly life-threatening bleeding complication is gastrointestinal bleeding from angiodysplasia. ${ }^{12}$ It is most common in elderly patients with type 2 or 3 VWD. ${ }^{12,13}$ The presenting patient had history of gastrointestinal bleeding in the form of haematemesis and melena. During admission, the child was well thriving, moderately pale, multiple bruises over extremities, and she had no active bleeding from any site.

The diagnosis of VWD is based on history of bleeding, a family history of bleeding, or both, in combination with laboratory tests showing abnormalities in VWF, factor VIII, or both. Most patient may have a family history of bleeding. But our patient didn't have history of such type of illness among her family members. Though there are no reliable screening test for VWD, patient with significant bleeding may present with anaemia. Some patient with Type 2B VWD or platelet-type, pseudo-VWD may have thrombocytopenia. ${ }^{2}$ Our patient had low haemoglobin concentration $(\mathrm{Hb} \% 8.8 \mathrm{~g} / \mathrm{dl})$, and platelet count was normal. Platelet function analysis has been considered as a screening test for VWD; but suboptimal sensitivity and specificity render results difficult to interpret. ${ }^{2}$ Bleeding time is similarly unreliable in diagnosis of VWD. Our patient had bleeding time of 10 minutes and clotting time of 15 minutes. Unfortunately, there is no single test that can reliably diagnose VWD. Therefore, a panel of tests is usually required. These include VWF:Ag which measures the total amount of VWF protein present, and VWF activity, which is typically performed using the ristocetin cofactor activity assay (VWF:RCo) and provides a measure of the amount of functional VWF. ${ }^{2}$ Our patient had VWF:Ag $1 \%$ that confirmed a severe Type 3 VWD. Genetic diagnosis is not typically performed, partly because of the large size of VWF gene and the large number of benign sequence variations. We also did bone marrow study and upper $\mathrm{Gl}$ endoscopy to exclude other causes of GI bleeding and unexplained anaemia of the patient.

Treatment of VWD depends on the type of VWD. In general, Type 1 VWD patient may be treated with desmopressin which increase the amount of circulating VWF by release from storage. Treatment of Type 2 \& 3 VWD requires VWF containing concentrate similar to the treatment of haemophilia. ${ }^{1}$ After consultation with Paediatric Haematology\& Oncology Department the child was treated and discharged with recommendation of sedentary lifestyle in combination with prophylactic therapy in minor surgery including dental work. Desmopressin nasal spray was advised when necessary (if bleeding occurs). Careful monitoring of VWF and FVIII levels is recommended to tailor treatment for surgeries and major trauma. For all types of VWD, adjunct therapy should be considered when possible, such as the use of antifibrinolytics for oral surgery or hormonal treatment for menorrhagia. Alternate treatment strategies should also be considered, particularly for difficult symptoms or severe VWD. Hormonal therapy for women with menorrhagia, although not specific to VWD, can be very helpful in managing symptoms and improving quality of life. Local treatment of epistaxis, such as nasal cautery or packing, may be helpful in some circumstances. Iron therapy for patients with iron-deficiency anaemia may also be required. ${ }^{1}$

\section{Conclusion}

Though VWD is the most common inherited bleeding disorder even diagnosis is sometimes difficult. The aim of this work is to show that VWD can be rarely present with UGI bleeding. So, physicians should always consider coagulation disorders including VWD during the assessment of any patient with recurrent $\mathrm{Gl}$ bleeding.

\section{References}

1. Sibal A, Gopalan S, Kapoor A, Bhatia V. Textbook of pediatric gastroenterology, hepatology and nutrition. New Delhi : JP Medical Ltd; 2015.

2. Kliegman R, Stanton B, St. Geme J, Schor N, Nelson W. Nelson textbook of pediatrics. Philadelpha, PA: Elsevier; 2016. 
3. Goswami M, Thakkar D. Von Willebrand disease - broadening our vision: a pediatric case report with dental considerations. J Dent Specialities. 2017;5:70-3.

4. Bozkurt M, Barut M. Von Willebrand's disease diagnosed after hemorrhage following hysteroscopic myoma resection and endometrial band excision. Proceedings in Obstetrics and Gynecology. 2013;3:1-5.

5. Sharanamma B, Siddarth G, Basavaraj TB, Pankaj J. Von Willebrand Disease - Report of a case and review of literature. J. Int Oral Health. 2011;3:23-7.

6. Puri MS, Gaikwad VA, Bora PM, Tyagi SA, Singhania SS. A rare case of von willebrand disease as a cause of menorrhagia since menarche: A case report from western India with brief review. Bioinfo Publications. 2012;3: 37-8.

7. Cook R, Marques M. An Adolescent with a History of Menorrhagia. LabMedicine. 2009; 40:271-3.

8. Gupta P, Jan SM, Behal R, Nazir G. Gingival enlargement in von Willebrand disease: A case report. Journal of Indian Society of Periodontology. 2014;18:390-4.

9. Echahdi $\mathrm{H}$, Hasbaoui B, Khorassani M, Agadr A, Khattab M. Von Willebrand's disease: case report and review of literature. Pan African Medical Journal. 2017; 27.

10. Leebeek F, Eikenboom J. Von Willebrand's Disease. New England Journal of Medicine. 2016;375:2067-80.

11. Hartholt R, van Velzen A, Peyron I, ten Brinke A, Fijnvandraat K, Voorberg J. To serve and protect: The modulatory role of von Willebrand factor on factor VIII immunogenicity. Blood Reviews. 2017;31:339-47.

12. van Galen KPM, Timmer MA, de Kleijn P, Fischer K, Foppen W, Schutgens REG et al. Joint assessment in von Willebrand disease: Validation of the Haemophilia Joint Health score and Haemophilia Activities List. Thrombosis and Haemostasis. 2017;117:1465-70.

13. Joshi N, Kopec A, Ray J, Cline-Fedewa H, Groeneveld D, Lisman T et al. Von Willebrand factor deficiency reduces liver fibrosis in mice. Toxicology and Applied Pharmacology. 2017; 328:54-9. 